much to enhance the position of the Department. Work in the Department is centred on nuclear, solidstate, low-temperature, shower and field theory.

An original gift of $£ A .50,000$ by Dr. G. B. S. Falkiner was used to establish the F. B. S. Falkiner Nuclear Research Laboratory in memory of his father. This Laboratory, with twenty scientists, has been working during the past six years on various aspects of cosmic radiation and forms the basis of the new department. At present there are two large groups. One is specializing in the use of nuclear emulsions for the study of the primary cosmic radiation and high energy jets; the second group is concerned with a large-scale study of extensive air showers.

Considerable expansion of the efforts within the Department have taken place since the appointment of Prof. C. B. A. McCusker as leader of the cosmicray team of workers. Further expansion in the work of both the emulsion and extensive air shower groups is taking place at present.

The equipment from the large Culham air shower experiment which was run by the Harwell group in England arrived in Australia earlier during the year and has now been integrated into the former air shower experiment in Sydney. The American Air Force in Texas has recently flown $£ 5,000$ 's worth of nuclear emulsion for the emulsion group to a height of $126,000 \mathrm{ft}$. Balloons of $3,000,000 \mathrm{eu}$. ft. capacity were used. The flights were made gratis. The emulsion is to be processed in Chicago using the large developing facilities available in Prof. Marcel Schein's group.

The Wills Thermonuclear Department is a new one and has been made possible by a gift of $£ A .50,000$ from W. D. and H. O. Wills (Australia) Ltd. in Sydney. The gift will be used for providing some of the initial research equipment required by the Department. The creation of a thermonuclear department in the School is in keeping with recent developments overseas in the thermonuclear field, where there has been a marked tendency for the research effort to swing back from large-scale engineering attacks to work of a more fundamental nature and on a smaller scale. It will also allow Australia to partake from an early stage in this exciting field of research.

The appointment of Prof. C. N. Watson Munro, chief scientist of the Australian Atomic Energy Commission, to the chair of thermonuclear physics is in keeping with his experience in the nuclear field. He was one of the pioneers in the nuclear reactor field and it is his wish now to try to pioneer some of the work in the thermonuclear field.

The Chatterton Astronomy Department will have two groups. One group will use a 40-in. reflecting telescope with auxiliary photoelectric equipment to consider problems in astronomy, the interpretation of which relies heavily upon physics. The contract for the instrument will be placed during 1960 and the instrument should be installed during 1961. Total initial cost of the project will be around $£ A .100,000$. The site for the instrument has still to be determined, but is highly likely to be situated at a joint Mount Stromlo-Sydney School of Physics field station. A survey for the site is at present being carried out by the Mount Stromlo group.

The second group will in the first instance be concerned with work using an intensity interferometer (at a later date a general interferometry group will be built up). This instrument, the first of its kind to be built, is based upon the work of two British scientists, Mr. Hanbury Brown of the University of Manchester and Dr. R. Q. Twiss, who is a member of staff of the Chatterton Astronomy Department. The instrument will be used for measuring the temperature and diameter of stars - both very important quantities in astrophysics.

The contract for the instrument has been let in England and the interferometer will arrive in Australia towards the end of 1960 . The site for the field station at which the instrument will be erected is likely to be at Narrabri, New South Wales-about 370 miles north-west of Sydney. Initial cost of the project will be in the neighbourhood of £A.175,000. Initial funds for work in the Astronomy Department were provided by a grant of $£$ A.50,000 from Mr. Stan Chatterton, a prominent member of the Nuclear Research Foundation within the University of Sydney.

It will be noted that the five new departments within the School of Physics form an integrated research effort. The work of all the departments is closely linked and has been chosen with that purpose in mind. It should allow Australia to make an everincreasing contribution to furthering new knowledge in these fields.

The Nuclear Research Foundation within the University of Sydney has been responsible for the provision of the major funds for the research effort in the School of Physics. A sum approaching $£ A .1,000,000$ has been raised in various ways during the past six years.

\title{
OBITUARY
}

\section{Dr. H. H. Potter}

THE death is announced of Dr. Harold Herbert Potter, lecturer in physics in the University of Bristol from 1924 until his retirement last summer owing to ill-health. Dr. Potter was a graduate of Bristol, who spent four years at King's College, London, as the holder of an 1851 Exhibition before returning to Bristol. At King's College, under O. W. Richardson, he carried out accurate measurements on the proportionality between mass and weight, and showed that tho ratio is independent of the nature of the material within an experimental error of fifteen parts in a million.
To workers in the field of magnetism, Potter was well known for his investigations in Bristol in the field of ferromagnetics, both in collaboration with Sucksmith and independently between the two World Wars. This work included specific heat measurements through the Curie point; the magnetic properties of single crystals and their correlation with crystal structure, ineluding Heusler and other magnetic alloys; and the electrical resistivity of nickel and iron from $20^{\circ}$ to $1,200^{\circ} \mathrm{K}$.

As an experimenter, Potter's work was careful, reliable and important in its period; it will also stand the test of time. In addition, he was a keen, effective and popular teacher of undergraduates.

He leaves a widow but no children. 